
THROMBOLYSED PATIENTS

Authors: M. Arsenijević1 ${ }^{\text {, I. Berisavac }}{ }^{1,2}$, P. Stanarčević ${ }^{1,2}$, T. Švabićn ${ }^{2}$, V. Pađen ${ }^{2}$, M. Stefanović Budimkić2, A. Radivojević1 ${ }^{1}$ M. Ercegovac ${ }^{1,2}$, D. Jovanović1,2

${ }^{1}$ Faculty of Medicine, University of Belgrade, Belgrade, Serbia.

${ }^{2}$ Department of Emergency neurology, Neurology Clinic- Clinical centre of Serbia, Belgrade, Serbia.

Background and Aim:

The National Institutes of Health Stroke Scale (NIHSS) is a tool that objectively quantifies the impairment caused by a stroke ${ }^{1}$. Its baseline score strongly predicts outcome in patients with acute ischemic stroke (AIS) ${ }^{2}$.

The aim of our study was to determine risk factors for severe stroke in thrombolysed patients.
Patients and Methods:

A total of 514 thrombolysed patients were classified according to the baseline NIHSS score into two groups- patients with baseline NIHSS score $\leq 15(n=346)$ and patients with baseline NIHSS score $>15$ ( $n=168)$.

Statistics: T-test, Mann-Whitney test and $\chi 2$ test were used for univariate analysis. Multivariate logistic regression was used to assessing the impact of those factors that showed significant in a previous univariate analysis.

Results:

Table 1. The influence of the basic demographic characteristics and the presence of risk factors on the stroke severity in the thrombolysed patients

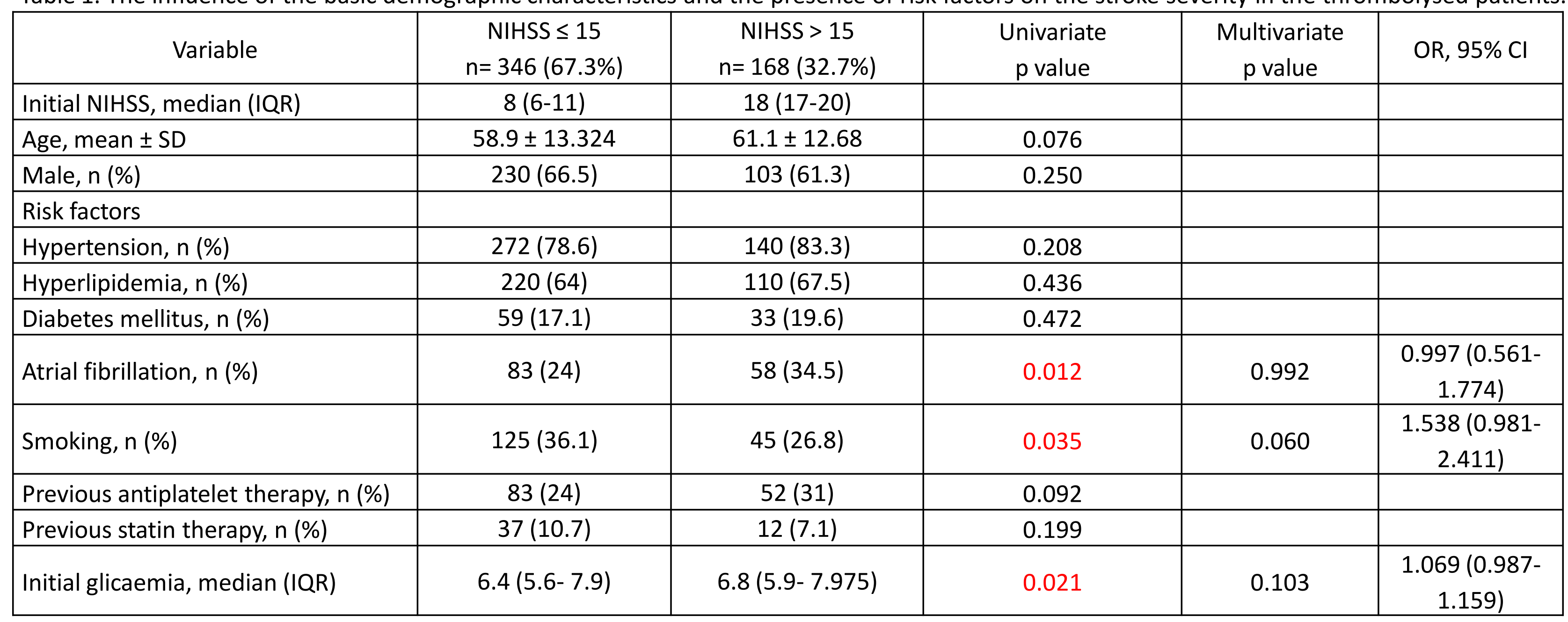

Table 2. The influence of the involvement of the carotid vascular area and left hemisphere on the stroke severity in the thrombolysed patients.

\begin{tabular}{|l|c|c|c|c|c|}
\hline Variable & $\begin{array}{c}\text { NIHSS } \leq 15 \\
\mathrm{n}=346 \\
(67.3 \%)\end{array}$ & $\begin{array}{c}\text { NIHSS }>15 \\
\mathrm{n}=168 \\
(32.7 \%)\end{array}$ & $\begin{array}{c}\text { Univariate } \\
\mathrm{p} \text { value }\end{array}$ & $\begin{array}{c}\text { Multivariate } \\
\mathrm{p} \text { value }\end{array}$ & $\begin{array}{c}\text { OR, } \\
95 \% \mathrm{Cl}\end{array}$ \\
\hline $\begin{array}{l}\text { Carotid } \\
\text { vascular area, } \\
\mathrm{n}(\%)\end{array}$ & $266(76.9)$ & $156(92.9)$ & $<0.001$ & 0.001 & $\begin{array}{c}3.248 \\
(1.578- \\
6.686)\end{array}$ \\
\hline $\begin{array}{l}\text { Left } \\
\text { hemisphere, } \\
\mathrm{n}(\%)\end{array}$ & $138(39.9)$ & $97(57.7)$ & $<0.001$ & 0.058 & $\begin{array}{c}0.667 \\
(0.439- \\
1.013)\end{array}$ \\
\hline
\end{tabular}

Table 3. The influence of the time consumption on the stroke severity in the thrombolysed patients.

\begin{tabular}{|l|c|c|c|}
\hline Variable & $\begin{array}{c}\text { NIHSS } \leq 15 \\
\mathrm{n}=346(67.3 \%)\end{array}$ & $\begin{array}{c}\text { NIHSS }>15 \\
\mathrm{n}=168(32.7 \%)\end{array}$ & $\begin{array}{c}\text { Univariate } \\
\mathrm{p} \text { value }\end{array}$ \\
\hline $\begin{array}{l}\text { Time to door, } \\
\text { mean } \pm \text { SD }\end{array}$ & $80.81 \pm 44.41$ & $75.6 \pm 38.53$ & 0.399 \\
\hline $\begin{array}{l}\text { Time to exam, } \\
\text { mean } \pm \text { SD }\end{array}$ & $90.23 \pm 45.25$ & $85.05 \pm 37.55$ & 0.420 \\
\hline
\end{tabular}

Table 4. The influence of the etiology according to the TOAST classification ${ }^{3}$ on the stroke severity in the thrombolysed patients.

\begin{tabular}{|c|c|c|c|c|c|}
\hline Variable & $\begin{array}{c}\text { NIHSS } \leq 15 \\
n=346 \\
(67.3 \%)\end{array}$ & $\begin{array}{c}\text { NIHSS }>15 \\
n=168 \\
(32.7 \%)\end{array}$ & $\begin{array}{c}\text { Univariate } \\
p \text { value }\end{array}$ & $\begin{array}{c}\text { Multivariate } \\
\text { p value }\end{array}$ & $\begin{array}{c}\text { OR, } \\
95 \% \mathrm{Cl}\end{array}$ \\
\hline LAA, n (\%) & 63 (18.3) & $34(20.2)$ & \multirow{5}{*}{$<0.001$} & $<0.001$ & $\begin{array}{c}7.736 \\
(2.527- \\
23.679)\end{array}$ \\
\hline $\begin{array}{l}\text { Embolism, } \\
\mathrm{n}(\%)\end{array}$ & $87(25.2)$ & 65 (38.7) & & $<0.001$ & $\begin{array}{c}7.986 \\
(2.526- \\
25.244)\end{array}$ \\
\hline Other, n (\%) & 35 (10.1) & $10(6.0)$ & & 0.001 & $\begin{array}{c}4.657 \\
(1.311- \\
16.547)\end{array}$ \\
\hline $\begin{array}{l}\text { Undetermined, } \\
\mathrm{n}(\%)\end{array}$ & $109(31.6)$ & $55(32.7)$ & & 0.017 & $\begin{array}{l}6.786 \\
(2.297- \\
20.042\end{array}$ \\
\hline SAO, n (\%) & $51(14.8)$ & $4(2.4)$ & & Referent & tegory \\
\hline
\end{tabular}

LAA- large artery atherosclerosis, SAO- small artery occlusion

\title{
Conclusion
}

Predictors for the development of severe AIS in thrombolysed patients are- involvement of

the carotid vascular area, large arteries atherosclerosis, cardioembolism, other determined

\section{and undetermined etiologies.}

References: 1. Brott T, Adams HP, Jr, Olinger CP, et al. Measurements of acute cerebral infarction: a clinical examination scale. Stroke. 1989;20:864-870. 2. Adams HP Jr, Davis PH, Leira EC, et al. Baseline NIH Stroke Scale score strongly predicts outcome after stroke : A report of the Trial of Org 10172 in Acute Stroke Treatment (TOAST). Neurology. 1999 Jul 13;53(1):126-31. 3. Adams HP, Bendixen BH, Kappelle LJ, Biller J, Love BB, Gordon DL, Marsh EE. Classification of subtype of acute ischemic stroke.Definitions for use in a multicenter clinical trial.TOAST.Trial of Org 10172 in Acute Stroke Treatment. Stroke 1993; 24(1): 35-41. 